\title{
The Sensitivity Analysis of a Lake Ecosystem with the Conditional Nonlinear Optimal Perturbation Method
}

\author{
Bo Wang, ${ }^{1,2}$ Peijun Zhang, ${ }^{3}$ Zhenhua Huo, ${ }^{2}$ and Qianqian $\mathbf{Q i}^{2}$ \\ ${ }^{1}$ Institute of Applied Mathematics, Henan University, Henan Kaifeng 475004, China \\ ${ }^{2}$ College of Mathematics and Information Science, Henan University, Henan Kaifeng 475004, China \\ ${ }^{3}$ Key Laboratory of Ocean Circulation and Wave, Institute of Oceanology, Chinese Academy of Sciences, Qingdao 266071, China
}

Correspondence should be addressed to Bo Wang, wangbo_sdu@163.com

Received 4 April 2012; Revised 6 June 2012; Accepted 23 June 2012

Academic Editor: Wansuo Duan

Copyright (C) 2012 Bo Wang et al. This is an open access article distributed under the Creative Commons Attribution License, which permits unrestricted use, distribution, and reproduction in any medium, provided the original work is properly cited.

\begin{abstract}
The instability and sensitivity of a lake ecosystem to the finite-amplitude perturbations related to the initial condition and the parameter correspondingly are studied. The CNOP-I and CNOP-P methods are adopted to investigate this nonlinear system. The numerical results with CNOP-I method show that the lake ecosystem can be nonlinearly unstable with finite-amplitude initial perturbations when the nutrient loading rate is between the two bifurcation points. A large enough finite amplitude initial perturbation, that is, CNOP-I, can induce a transition from an oligotrophic (eutrophic) state to an eutrophic (oligotrophic) state. With CNOP-P method, it is shown that the lake ecosystem can be transformed from an oligotrophic (eutrophic) state to an eutrophic (oligotrophic) state with a large enough finite amplitude parameter perturbation, that is, CNOP-P, no matter how large the nutrient loading rate is.
\end{abstract}

\section{Introduction}

Lake eutrophication is a serious economic, recreational, environmental, and aesthetic problem that affects every lake of the world. According to one of the recent studies, it is not nitrogen but phosphorus that should be reduced to mitigate eutrophication [1]. The relationship between the release of nutrients and eutrophication has been revealed [2]. For different nutrient lake sediments, some studies show the impact of organic matters on the release mechanism of phosphorus [3]. Also, several studies imply that the change of the phosphorus concentration is relevant to the variations of ecological parameter. Some studies investigate the effects of seasonal variations in ecological parameters, such as the numbers of daphnia and the biomass of the algae $[4,5]$.

There exist multiple equilibrium states in the lake ecosystem. In some conditions, the change of the external parameter can cause the ecosystem transition [6], which may be a transition between the clear, macrophyte-dominated state and the turbid state dominated by phytoplankton in a lake ecosystem.

In this paper, for a simple model which is developed by Carpenter et al. [7], we investigate whether and how transitions between the different equilibrium states happen. Here, the approach of conditional nonlinear optimal perturbation related to the initial condition and the parameter (CNOP-I and CNOP-P) is adopted to investigate the nonlinear instability and sensitivity of the lake eutrophication. The CNOP method is proposed and extended by $\mathrm{Mu}$ et al. $[8,9]$. The method has been used to study ENSO by Duan et al. [10-18], the response of a grassland ecosystem to the climate change [19-22], the sensitivity, and nonlinearity analysis of a lake ecosystem model [23]. All the former work demonstrates that CNOP method is very useful in studying the predictability, sensitivity and nonlinear stability analysis for nonlinear system.

In the next section, the model and the methods are described; in Section 3, with the CNOP-I and CNOP-P method, the instability and sensitivity analysis of the lake ecosystem is given; in the last section, we give the summary.

\section{The Model and the Methods}

2.1. The Model. We consider a lake eutrophication model of Carpenter et al. [7], which describes the phosphorus cycle 
in lakes and has one dimensionless dynamical variable: $p$, which represents the concentration of nutrients, especially the phosphorus. The existence of multiequilibrium states of this model has been revealed and studied by Guttal and Jayaprakash [24]. The model is as follows:

$$
\frac{d p}{d t}=c-s p+r \frac{p^{q}}{p_{0}^{q}+p^{q}}
$$

where the term $c$ is the nutrient loading rate, which is the control parameter of the model; $s$ is the rate of phosphorus's loss per unite time, when we consider that the loss process only includes sedimentation, then its value is 1.0; $r$ is the maximum rate of nutrient recycling, and under the condition that the phosphorus can be recycled completely from sediments or by consumers, its value is $1.0 ; p_{0}$ is the concentration of nutrients when the system's cycle utilization rates achieve half, here, its value is $1.0 ; q$ is the Hill coefficient, the value of which is 8.0. All the values of these terms are consistent with [24].

Here, we adopt a fourth-order Runge-Kutta scheme with a time step $d t=0.1$, which represents 0.1 year, and integrate the model (1) with the initial values and the corresponding nutrient loading rate to obtain the solution numerically. With the Spectral Projected Gradient (SPG) method and the optimal time $T=30$, that is, three years, the CNOP-Is and CNOP-Ps are obtained numerically.

2.2. CNOP Method. Assume that a dynamical model is as follows:

$$
\frac{\partial U}{\partial t}=F(U, P), \quad(x, t) \in \Omega \times[0, \tau],
$$

where $U(x, t)=\left(U_{1}(x, t), U_{2}(x, t), \ldots, U_{n}(x, t)\right)$ is the state variable, and $U_{0}$ is the initial state; $(x, t) \in \Omega \times[0, \tau], \Omega$ is a domain in $R^{n}, x=\left(x_{1}, x_{2}, \ldots, x_{n}\right) \in \Omega$; and $P$ represents the model parameter which is independent of time t.F is a nonlinear differential operator. Assume that the dynamical system equations (2) and the initial state are known exactly, then the future state can be determined by integrating (2). The state vector $U$ at time $\tau$ is given by

$$
U(\tau)=M_{\tau}(P)\left(U_{0}\right) .
$$

Here, $M_{\tau}$ is the propagator of (2) from the time 0 to the time $\tau$ in the future.

Now we explore the situation that there exists both initial perturbation and parameter perturbation in the model. Then we have

$$
U(\tau)+u\left(u_{0}, p^{\prime} ; \tau\right)=M\left(P+P^{\prime}\right)\left(U_{0}+u_{0}\right) .
$$

Here $u\left(u_{0}, p^{\prime} ; \tau\right)$ is the deviation from the reference state $U(\tau)$ caused by the combined perturbation $\left(u_{0}, p^{\prime}\right)$.

A nonlinear optimization problem is defined as follows:

$$
\begin{gathered}
J\left(u_{0}, p^{\prime}\right)=\left\|M_{\tau}\left(p+p^{\prime}\right)\left(U_{0}+u_{0}\right)-M_{\tau}(p)\left(U_{0}\right)\right\|, \\
J\left(u_{0 \delta}, p_{\sigma}^{\prime}\right)=\max _{u_{0} \in c_{\delta}, p^{\prime} \in c_{\sigma}} J\left(u_{0}, p^{\prime}\right),
\end{gathered}
$$

where $u_{0} \in c_{\delta}$ and $p^{\prime} \in \mathcal{c}_{\sigma}$ are the constraint conditions of the initial perturbations and parameter perturbations, respectively. The combined perturbation $\left(u_{0 \delta}, p_{\sigma}^{\prime}\right)$ is called CNOP. So, for the given constraints, CNOP is the optimal combined mode of initial perturbations and parameter perturbations, which induces the largest deviation from the reference state at time $\tau$.

Particularly, if only considering the initial perturbation, namely, $p^{\prime}=0$, then the initial perturbation $u_{0 \delta}$ which satisfies the optimization problem is called CNOP-I; if only considering the parameter perturbations, namely, $u_{0}=0$, then the parameter perturbation $p_{\sigma}^{\prime}$ which satisfies the optimization problem is called CNOP-P.

\section{The Nonlinear Instability and the Sensitivity Analysis}

For the certain values of the parameter, the model has various equilibrium states and there are two bifurcation points: $c_{1}$ and $c_{2}$. Figure 1 shows the equilibrium states under the different values of the nutrient loading rate $c$. From Figure 1, we can see that for $c<c_{1}$, there exists only one linearly stable oligotrophic equilibrium state (OES, solid line); when $c_{1} \leq c \leq c_{2}$, there are one linearly unstable equilibrium state (dashed line) and two linearly stable states, one is an OES and the other is an eutrophic equilibrium state (EES); in the case of $c>c_{2}$, there just exists one linearly stable EES. This conclusion has been revealed by others [24] and we obtain Figure 1 by repeating their work based on the Lyapunov stability theory. In this paper, we will study the nonlinear instability of the linear stable equilibrium state.

3.1. The Impact of CNOP-I on the Lake Ecosystem. In this subsection, we will use the SPG method to obtain CNOP-I. What we should keep in mind is that the dynamical variable $p$ should be nonnegative and so the initial perturbation $p^{\prime}$ should satisfy both the relation: $p+p^{\prime} \geq 0$ and the restraint condition: $\left|p^{\prime}\right| \leq \delta$ : here $\delta$ is given arbitrary, but in general we think $0<\delta<2$ because of the restrain of the concentration of the phosphorus.

We get CNOP-Is corresponding to the parameter $c$ and the initial equilibrium states with different initial restraint conditions in the intervals $\left[0, c_{1}\right),\left[c_{1}, c_{2}\right]$, and $\left(c_{2}, 1\right]$. The results show that when $c<c_{1}$, the oligotrophic equilibrium state will not be transformed to an eutrophic state no matter how large the initial perturbation restraint $\delta$ is and so the lake ecosystem is nonlinearly stable; when $c_{1} \leq c \leq c_{2}$, the oligotrophic (eutrophic) equilibrium state can be transformed to an eutrophic (oligotrophic) equilibrium state with a large enough initial restraint $\delta$ and the lake ecosystem is nonlinearly unstable; when $c>c_{2}$, the system would transform from the eutrophic equilibrium state to the oligotrophic state no matter how large the initial perturbation restraint $\delta$ is and so the lake ecosystem is nonlinearly stable.

To illustrate the above results, we take OES A $(c=0.25$, $\left.p_{e}=0.250015\right)$ as an example in the interval $\left[0, c_{1}\right)$, OES $\mathrm{B}\left(c=0.55, p_{e}=0.559513\right)$ and $\operatorname{EES~C}\left(c=0.55, p_{e}=\right.$ $1.515268)$ as examples in interval $\left[c_{1}, c_{2}\right]$ and $\operatorname{EES~D}(c=$ 
TABLE 1: The CNOP-Is corresponding to different basic states.

\begin{tabular}{lccc}
\hline$\delta$ & $\begin{array}{c}\text { CNOP-I of OES A } \\
(c=0.25)\end{array}$ & $\delta$ & $\begin{array}{c}\text { CNOP-I of OES B } \\
(c=0.55)\end{array}$ \\
\hline 0.35 & 0.35 & 0.25 & 0.25 \\
0.45 & 0.45 & 0.35 & 0.35 \\
0.85 & 0.85 & 0.4 & 0.4 \\
1.0 & 1.0 & 0.5 & 0.5 \\
\hline$\delta$ & CNOP-I of EES C & $\delta$ & CNOP-I of EES D \\
& $(c=0.55)$ & & $(c=0.75)$ \\
\hline 0.35 & -0.35 & 0.5 & -0.5 \\
0.5 & -0.5 & 0.75 & -0.75 \\
0.75 & -0.75 & 1.5 & -1.5 \\
1.0 & -1.0 & 1.738138 & -1.738138 \\
\hline
\end{tabular}

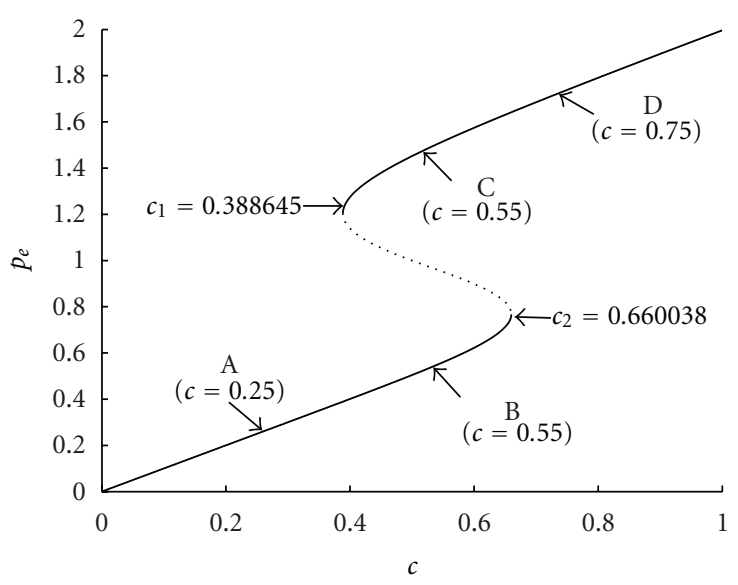

FIGURE 1: The equilibrium states versus the nutrient loading rate $c$, where $c_{1}$ and $c_{2}$ are the bifurcation points, the solid line refers to the linearly stable equilibrium states and the dashed line refers to the linearly unstable equilibrium states.

$\left.0.75, p_{e}=1.738138\right)$ as an example in interval $\left(c_{2}, 1\right]$. Then we take $p_{0}+\mathrm{CNOP}-\mathrm{I}$ as the initial state and $c$ as the parameter to integrate the model with the integral time $T=150$, that is, 15 years. The results are shown in Table 1 and Figure 2.

For the case of $c<c_{1}$, the conclusion can be yielded as that the ecosystem is nonlinearly stable and there are only OESs. In order to show the results, we take the case of $c=0.25$ as an example. From Table 1 and Figure 2(a), we can see that all the CNOP-Is are positive and they make the ecosystem recover back to the OES A. And the larger the amplitude $\delta$ is, the longer time will be used for the ecosystem to return to OES A. Here we give the physical mechanism: the low nutrient loading rate restrains the growth of the algae and the plankton, which would increase the amount of the dissolved oxygen in the water and improve the quality of the water, so the lake flows back to OES.

For OES B, from the Figure 2(b), we can see that when $\delta=0.25$ and $\delta=0.35$, the CNOP-Is cause the ecosystem to return to OES $\mathrm{B}$, and it takes much longer time to evolve to OES B for a larger amplitude $(\delta=0.35)$. When $\delta=0.4$ and $\delta=0.5$, the CNOP-Is causes a transition to EES C and the larger $\delta$ is, the shorter time it takes to shift to EES C $(\delta=0.5)$. Here we take the example of $\delta=0.5$ to explain the lake ecosystem's physical mechanism. On this occasion, the initial values satisfy that $d p / d t$ are positive. So at the beginning the phosphorus concentration from the lake increases. Then it makes the algae and the plankton increase quickly and causes reduction in the amount of dissolved oxygen in the lake. After dissolved oxygen's decreasing for some time, the fish and the other creatures begin to die. The massive deaths of aquatic creatures make their corpses fall down to the bottom of the lake and then these corpses are decomposed by the microbial. At the same time, it consumes a lot of dissolved oxygen and causes the dissolved oxygen declining fast. So the water quality will be deteriorated. After several years, the lake shifts to EES C.

Through Figure 2(c), we get the following results. In case of $\delta=0.75$ and $\delta=1.0$, the CNOP-Is cause the ecosystem to evolve to OES B. The larger $\delta$ is, the shorter time the ecosystem takes to develop to OES B. This shows that human's positive activities in short time such as the control of nutrition material into the water or the positive change of biological control and some natural factors can evolve the ecosystem from EES to OES. For $\delta=0.35$ and $\delta=0.5$, the ecosystems return to EES C eventually and it takes longer time to recover to EES $\mathrm{C}$ with $\delta=0.5$ than that with $\delta=$ 0.35 .

For other values of $c$ in $\left[c_{1}, c_{2}\right]$, we can also get similar conclusion with the corresponding equilibrium state plus the corresponding CNOP-Is as the initial values. So, we can get a conclusion that for $c$ in $\left[c_{1}, c_{2}\right]$, when the amplitude is large enough, certain initial perturbation can make the ecosystem evolve from the oligotrophic (eutrophic) equilibrium state to an eutrophic (oligotrophic) equilibrium state. The results show that when $c$ is in $\left[c_{1}, c_{2}\right]$, the ecosystem is nonlinearly unstable.

For the case of $c>c_{2}$, the EES is nonlinearly stable. In this case, one can find in Figure $2(\mathrm{~d})$ that, no matter how large $\delta$ is and whether the CNOP-Is make the initial values of the phosphorus concentration equal 0 , the ecosystem will never evolve to OES. So when the nutrient loading rate is high enough, the EES is nonlinearly stable. It shows that human's activities and nature factors cannot shift the ecosystem from EES to OES.

In addition, we find that the initial equilibrium state corresponding to the parameter $c$ in the bifurcation interval $\left[c_{1}, c_{2}\right]$ will be transformed with the critical initial restraint $\delta_{c}$, which means that the lake ecosystem is nonlinearly unstable and the initial equilibrium state will be transformed with the initial restraint $\delta \geq \delta_{c}$, and the lake ecosystem is nonlinearly stable and the initial equilibrium state will not be transformed with the initial restraint $\delta<\delta_{c}$. Here, we show the result in Figure 3.

In addition, to further study the nonlinear feature of the ecosystem, we calculate linear singular vectors (LSVs) of OESs and EESs and compare them with the corresponding CNOP-Is. The results indicate that CNOP-Is are consistent with LSVs for the same magnitude of initial perturbation restraint, as the lake model is simple with one dynamic variable. So, we guess that for simple one-variable dynamic 


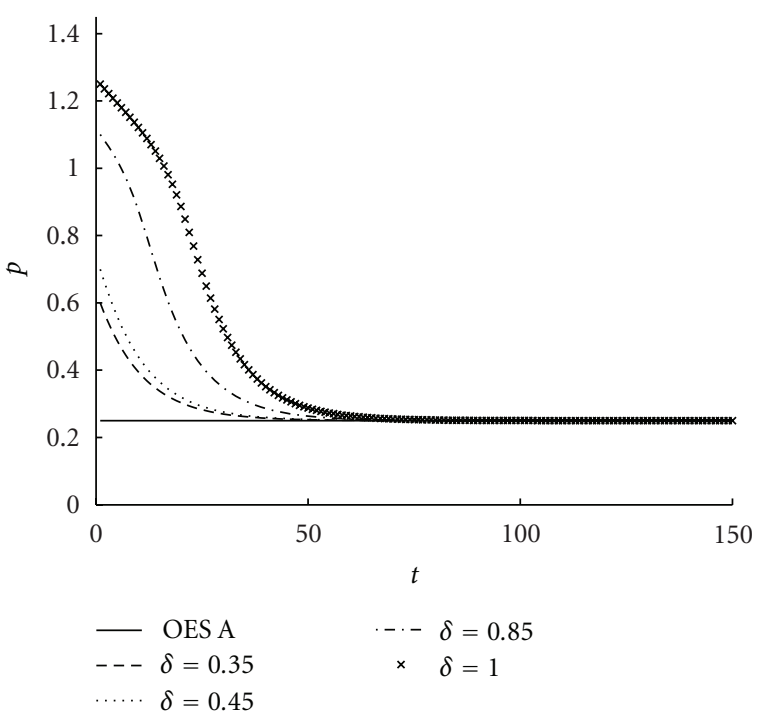

(a)

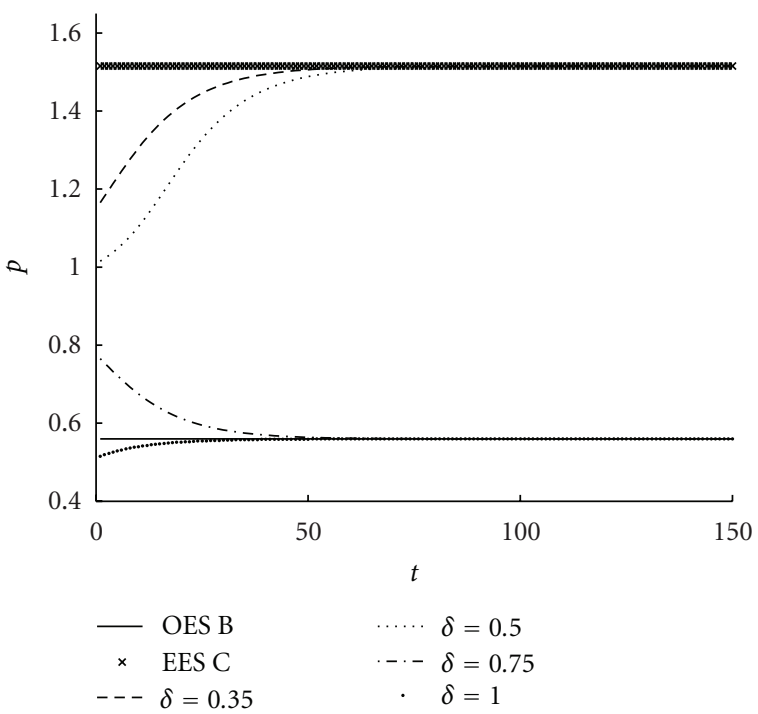

(c)

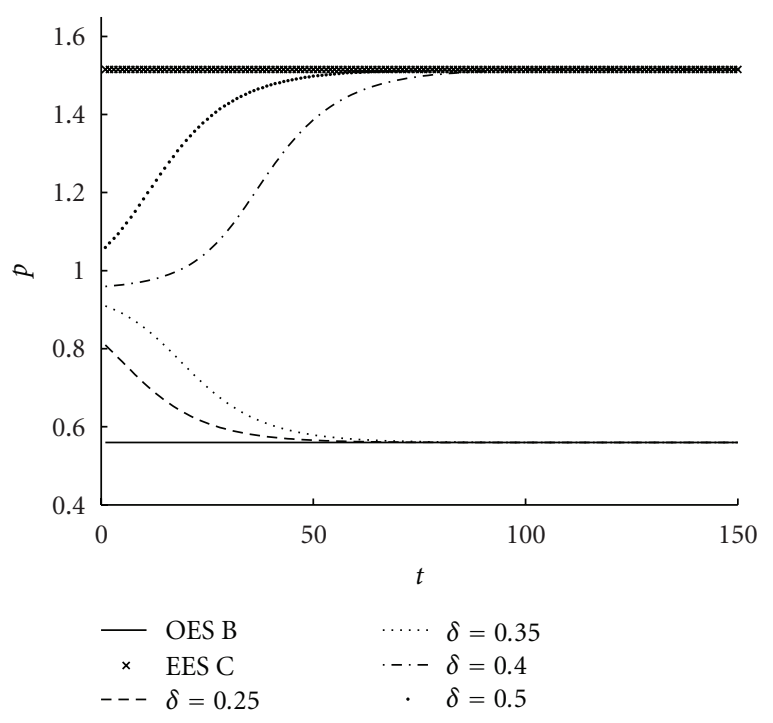

(b)

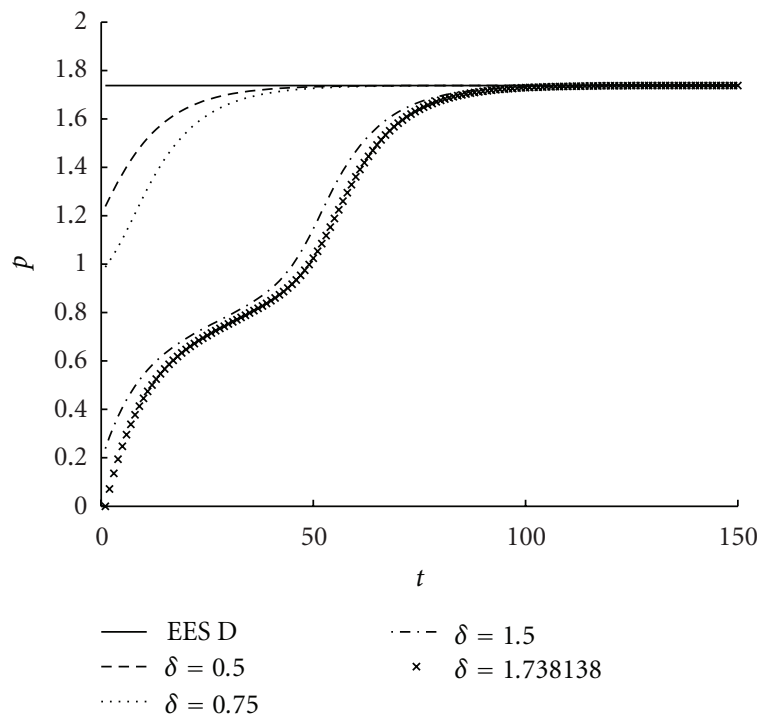

(d)

Figure 2: The 15-year nonlinear evolution of the lake ecosystem, with $p_{0}+\mathrm{CNOP}-\mathrm{I}$ as the initial condition and $c$ as the control parameter. (a) $c=0.25$, the basic state is OES A; (b) $c=0.55$, the basic state is OES B; (c) $c=0.55$, the basic state is EES C; (d) $c=0.75$, the basic state is EES D.

model, CNOP-Is may be consistent with LSVs for the same magnitude of initial perturbation restraint.

3.2. The Impact of CNOP-P on the Lake Ecosystem. In this subsection, we still use the SPG method to obtain CNOP-P. We know that the parameter $c$, the nutrient loading rate, belongs to the interval $[0,1]$ and so the parameter perturbation $c^{\prime}$ should satisfy both the relation: $0 \leq c+c^{\prime} \leq 1$ and the restraint condition: $\left|c^{\prime}\right| \leq \sigma$.

We get CNOP-Ps corresponding to the parameter $c$ and the initial equilibrium states with different parameter restraint conditions in the intervals $\left[0, c_{1}\right),\left[c_{1}, c_{2}\right]$, and $\left(c_{2}, 1\right]$. Finally, the results show that when $c<c_{2}$ and the initial equilibrium state is the oligotrophic state, if $\sigma \leq$ $c_{2}-c$, we get CNOP-P $=\sigma$ and the lake ecosystem will be transformed from an oligotrophic state to another oligotrophic state; if $c_{2}-c<\sigma<1-c$, we have CNOP-P $=\sigma$ and the lake ecosystem will be transformed from an oligotrophic state to an eutrophic state; with $\sigma \geq 1-c$, we get CNOP$\mathrm{P}=1-c$ and the lake ecosystem will be transformed from an oligotrophic state to an eutrophic state. When $c>c_{1}$ and the initial equilibrium state is the eutrophic state, if $\sigma \leq c-$ $c_{1}$, there is CNOP-P $=-\sigma$ and the ecosystem will be transformed from an eutrophic state to another eutrophic state; with the restraint condition $c-c_{1}<\sigma<c$, we have 


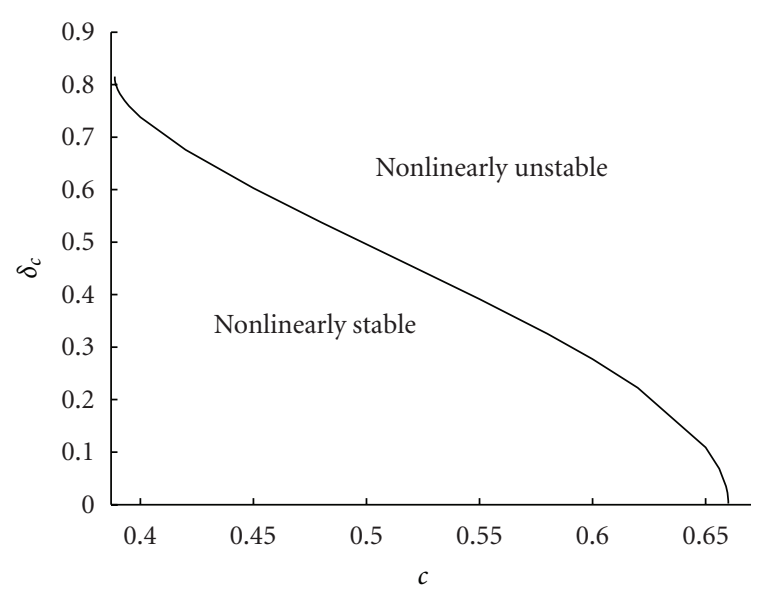

(a)

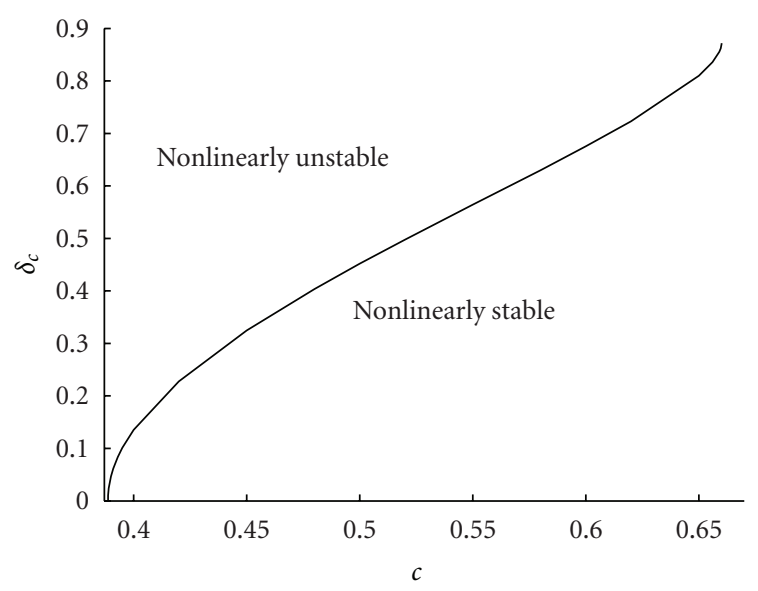

(b)

FIGURE 3: The critical value of $\delta_{c}$ versus the parameter, the nutrient loading rate, $c$. (a) for OES; (b) for EES.
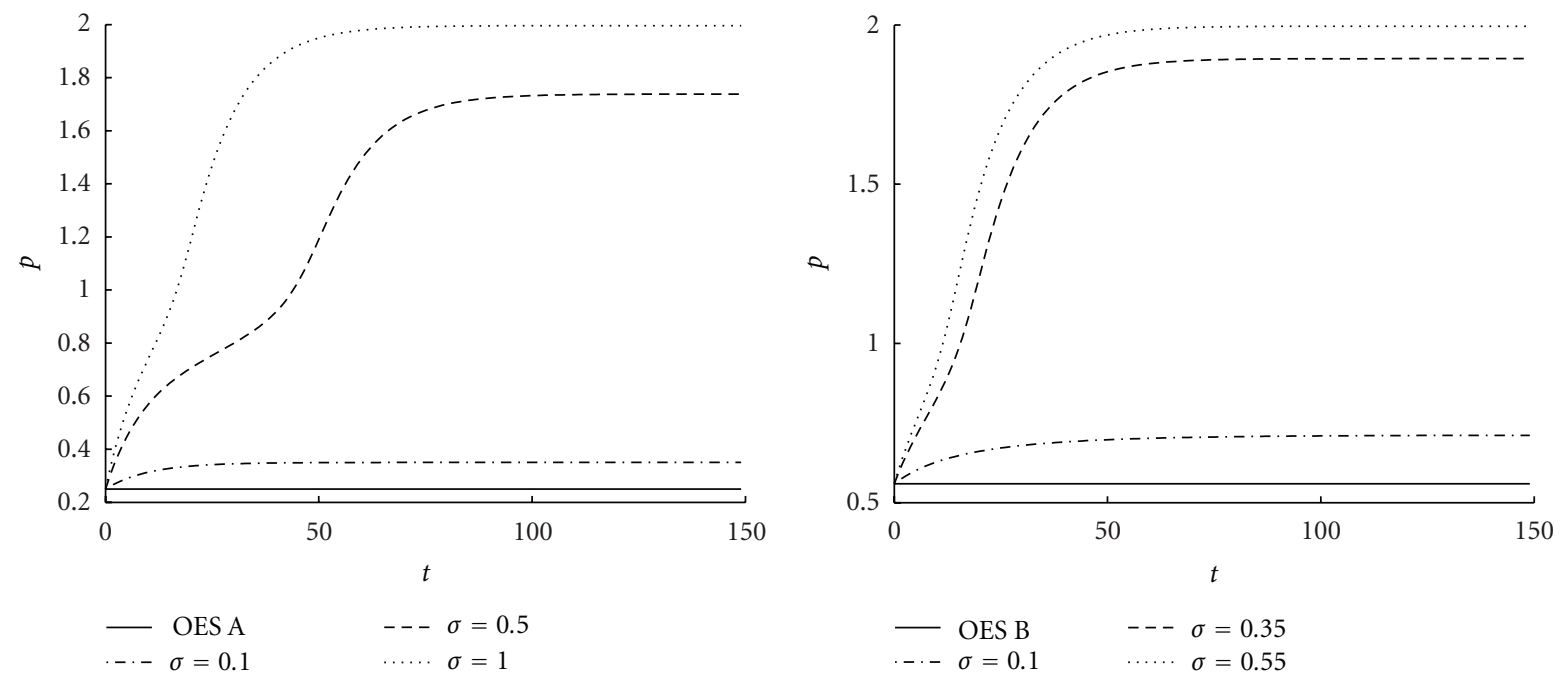

(a)
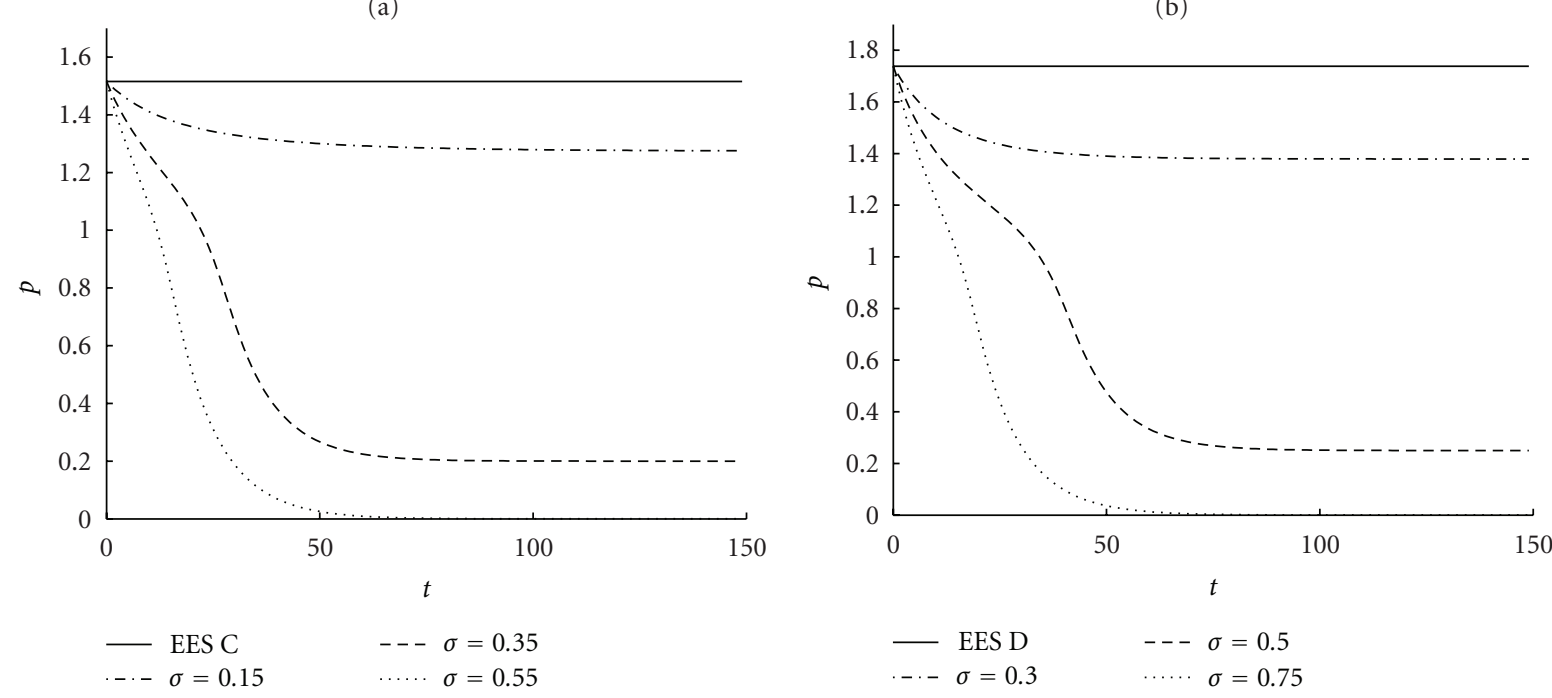

(c)

(d)

FIGURE 4: The 15-year nonlinear evolution of the lake ecosystem, with the basic state as the initial condition and $c+\mathrm{CNOP}-\mathrm{P}$ as the control parameter. (a) $c=0.25$, the basic state is OES A; (b) $c=0.55$, the basic state is OES B; (c) $c=0.55$, the basic state is EES C; (d) $c=0.75$, the basic state is EES D. 
TABLE 2: CNOP-Ps corresponding to different basic states.

\begin{tabular}{lccc}
\hline$\sigma$ & $\begin{array}{c}\text { CNOP-P of OES A } \\
(c=0.25)\end{array}$ & $\sigma$ & $\begin{array}{c}\text { CNOP-P of OES B } \\
(c=0.55)\end{array}$ \\
\hline 0.1 & 0.1 & 0.1 & 0.1 \\
0.5 & 0.5 & 0.35 & 0.35 \\
1.0 & 0.75 & 0.55 & 0.45 \\
\hline$\sigma$ & CNOP-P of EES C & $\sigma$ & CNOP-P of EES D \\
& $(c=0.55)$ & & $(c=0.75)$ \\
\hline 0.15 & -0.15 & 0.3 & -0.3 \\
0.35 & -0.35 & 0.5 & -0.5 \\
0.55 & -0.55 & 0.75 & -0.75 \\
\hline
\end{tabular}

CNOP-P $=-\sigma$ and the ecosystem will be transformed from an eutrophic state to an oligotrophic state; if $\sigma \geq c$, the CNOP-P will be equal to $-c$ and the ecosystem will be transformed from an eutrophic state to an oligotrophic state.

To illustrate the above results, we take OES $\mathrm{A}$ as an example in interval $\left[0, c_{1}\right)$, OES $\mathrm{B}$ and EES $\mathrm{C}$ as an example in interval $\left[c_{1}, c_{2}\right]$, and EES D as an example in interval $\left(c_{2}, 1\right]$. We take the equilibrium state as the initial state and $c+$ CNOP-P as the control parameter to integrate the model with the integral time $T=150$, that is, 15 years. The results are shown in Table 2 and Figure 4.

Now, we take the case of OES B as an example to explain the physical mechanism. For $\sigma=0.1$, when we plug $c+$ CNOP-P into the model, $d p / d t>0$ and the phosphorus concentration in the lake ecosystem increases at first. Then the algae and the phycophyta can increase to a large amount. As the nutrient loading rate is not large enough and the breeding of the hydrophyte will consume the phosphorus, the lake ecosystem is not transformed to be an eutrophic state at last. For $\sigma=0.35$ and $\sigma=0.55$, when we plug $c+$ CNOP-P into the model, $d p / d t>0$ and so the phosphorus concentration increases at first. Then the algae and the phycophyta begin to breed at a large amount. As the the breeding of the algae and the phycophyta will induce the dissolved oxygen in the lake and the nutrient loading rate is too large, the lake ecosystem is transformed to an eutrophic state at last.

\section{The Summary}

Through the above discussion, we can know that when the nutrient loading rate $c$ is between the two bifurcation points, the ecosystem can become nonlinearly unstable with a large enough CNOP-I, which can induce a transition from oligotrophic (eutrophic) state to eutrophic (oligotrophic) state; when $c<c_{1}$ and $c>c_{2}$, the ecosystem is nonlinearly stable and the ecosystem will not be transformed no matter how large the initial restraint $\delta$ is. The ecosystem can be transformed from an oligotrophic (eutrophic) state to an eutrophic (oligotrophic) state with a large enough CNOP-P no matter how large the initial nutrient loading rate is.

In our future work, we will try to compute CNOPs, and compare the impacts of CNOP-I, CNOP-P, the simple combination of CNOP-I and CNOP-P and CNOP on the lake ecosystem under the same initial constraint and parameter constraint.

\section{Acknowledgments}

This research was supported by the National Natural Science Foundation of China (Grant no. 40805020), the National Science Foundation of Henan Education Department (Grant no. 2010A100003), and the Natural Science Foundation of Henan Province (Grant no. 112300410054).

\section{References}

[1] H. J. Wang and H. Z. Wang, "Mitigation of lake eutrophication: loosen nitrogen control and focus on phosphorus abatement," Progress in Natural Science, vol. 19, no. 10, pp. 1445-1451, 2009.

[2] P. M. Nyenje, J. W. Foppen, S. Uhlenbrook, R. Kulabako, and A. Muwanga, "Eutrophication and nutrient release in urban areas of sub-Saharan Africa-a review," Science of the Total Environment, vol. 408, no. 3, pp. 447-455, 2010.

[3] S. R. Wang, X. C. Jin, H. C. Zhao, X. N. Zhou, and F. C. Wu, "Effects of organic matter on phosphorus release kinetics in different trophic lake sediments and application of transition state theory," Journal of Environmental Management, vol. 88, no. 4, pp. 845-852, 2008.

[4] M. Scheffer, S. Rinaldi, Y. A. Kuznetsov, and E. H. van Nes, "Seasonal dynamics of Daphnia and algae explained as a periodically forced predator-prey system," Oikos, vol. 80, no. 3, pp. 519-532, 1997.

[5] M. Scheffer and S. Rinaldi, "Minimal models of top-down control of phytoplankton," Freshwater Biology, vol. 45, no. 2, pp. 265-283, 2000.

[6] R. Portielje and D. T. van der Molen, "Relationships between eutrophication variables: from nutrient loading to transparency," Hydrobiologia, vol. 408-409, pp. 375-387, 1999.

[7] S. R. Carpenter, D. Ludwig, and W. A. Brock, "Management of eutrophication for lakes subject to potentially irreversible change," Ecological Applications, vol. 9, no. 3, pp. 751-771, 1999.

[8] M. Mu, W. S. Duan, and B. Wang, "Conditional nonlinear optimal perturbation and its applications," Nonlinear Processes in Geophysics, vol. 10, no. 6, pp. 493-501, 2003a.

[9] M. Mu, W. S. Duan, Q. Wang, and R. Zhang, "An extension of conditional nonlinear optimal perturbation approach and its applications," Nonlinear Processes in Geophysics, vol. 17, no. 2, pp. 211-220, 2010.

[10] M. Mu and W. S. Duan, "A new approach to studying ENSO predictability: conditional nonlinear optimal perturbation," Chinese Science Bulletin, vol. 48, no. 10, pp. 1045-1047, 2003 b.

[11] W. S. Duan, M. Mu, and B. Wang, "Conditional nonlinear optimal perturbations as the optimal precursors for El NinoSouthern Oscillation events," Journal of Geophysical Research D, vol. 109, Article ID D23105, 12 pages, 2004.

[12] W. S. Duan and M. Mu, "Investigating decadal variability of El Nino-Southern Oscillation asymmetry by conditional nonlinear optimal perturbation," Journal of Geophysical Research C, vol. 111, Article ID C07015, 8 pages, 2006.

[13] W. S. Duan, F. Xue, and M. Mu, "Investigating a nonlinear characteristic of El Niño events by conditional nonlinear optimal perturbation," Atmospheric Research, vol. 94, no. 1, pp. 10-18, 2009. 
[14] W. S. Duan, H. Xu, and M. Mu, "Decisive role of nonlinear temperature advection in El Niño and La Niña amplitude asymmetry," Journal of Geophysical Research C, vol. 113, no. 1, Article ID C01014, 2008.

[15] W. S. Duan and M. Mu, "Conditional nonlinear optimal perturbation: applications to stability, sensitivity, and predictability," Science in China, vol. 52, no. 7, pp. 883-906, 2009.

[16] W. S. Duan, X. C. Liu, K. Y. Zhu, and M. Mu, "Exploring the initial errors that cause a significant "spring predictability barrier" for El Niño events," Journal of Geophysical Research C, vol. 114, Article ID C04022, 12 pages, 2009.

[17] W. S. Duan and H. Y. Luo, "A new strategy for solving a class of constrained nonlinear optimization problems related to weather and climate predictability," Advances in Atmospheric Sciences, vol. 27, no. 4, pp. 741-749, 2010.

[18] W. S. Duan and R. Zhang, "Is model parameter error related to a significant spring predictability barrier for El Niño events? Results from a theoretical model," Advances in Atmospheric Sciences, vol. 27, no. 5, pp. 1003-1013, 2010.

[19] G. D. Sun and M. Mu, "Response of a grassland ecosystem to climate change in a theoretical model," Advances in Atmospheric Sciences, vol. 28, no. 6, pp. 1266-1278, 2011.

[20] B. Wang, J. P. Wang, Z. H. Huo, P. J. Zhang, and Q. Wang, "Application of conditional nonlinear optimal perturbation method in a theoretical grassland ecosystem," Chinese Quarterly Journal of Mathematics, vol. 25, no. 3, pp. 422-429, 2010.

[21] B. Wang, Z. H. Huo, P. J. Zhang, and Q. Wang, "Impacts of human activities on the transition between grassland and desert in a simplified theoretical grass field ecosystem," in Climate Change and Ecosystem Response, pp. 204-207, 2011.

[22] M. Mu and B. Wang, "Nonlinear instability and sensitivity of a theoretical grassland ecosystem to finite-amplitude perturbations," Nonlinear Processes in Geophysics, vol. 14, no. 4, pp. 409-423, 2007.

[23] B. Wang, P. J. Zhang, Z. H. Huo, and Q. Wang, "Application of conditional nonlinear optimal perturbation in study of lake eutrophication," in Climate Change and Ecosystem Response, pp. 426-429, 2011.

[24] V. Guttal and C. Jayaprakash, "Impact of noise on bistable ecological systems," Ecological Modelling, vol. 201, no. 3-4, pp. 420-428, 2007. 

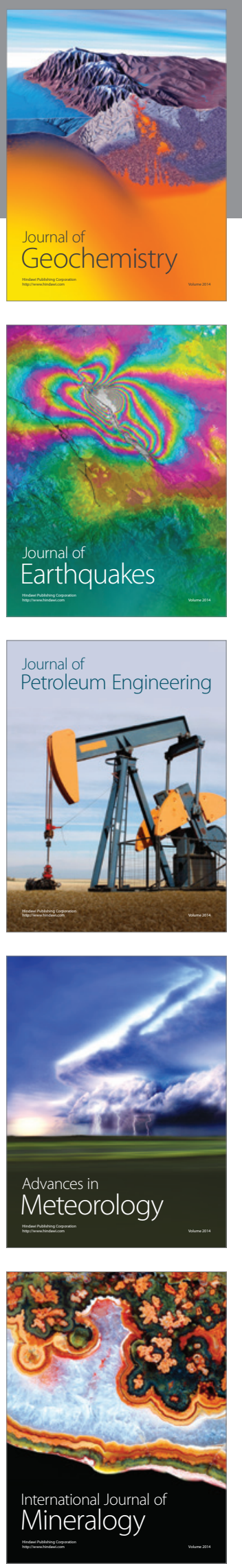
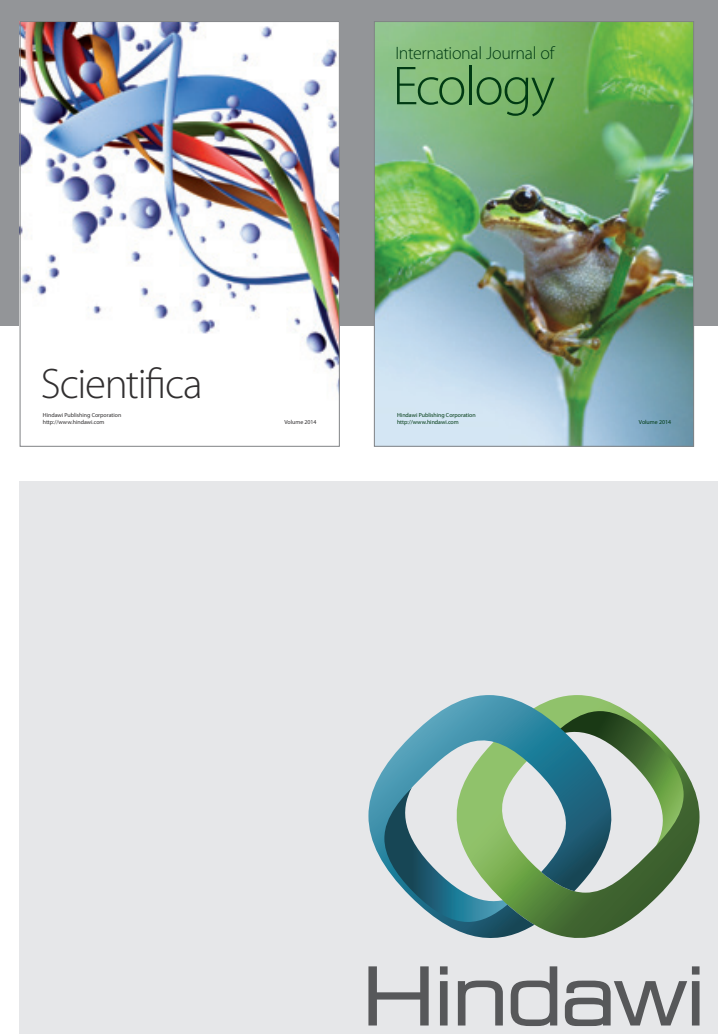

Submit your manuscripts at http://www.hindawi.com
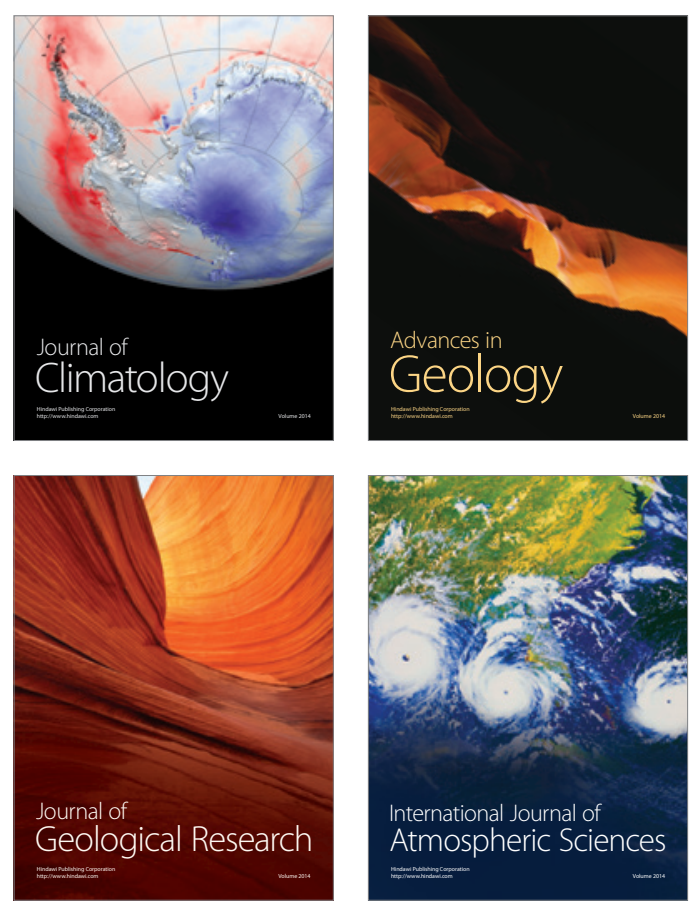
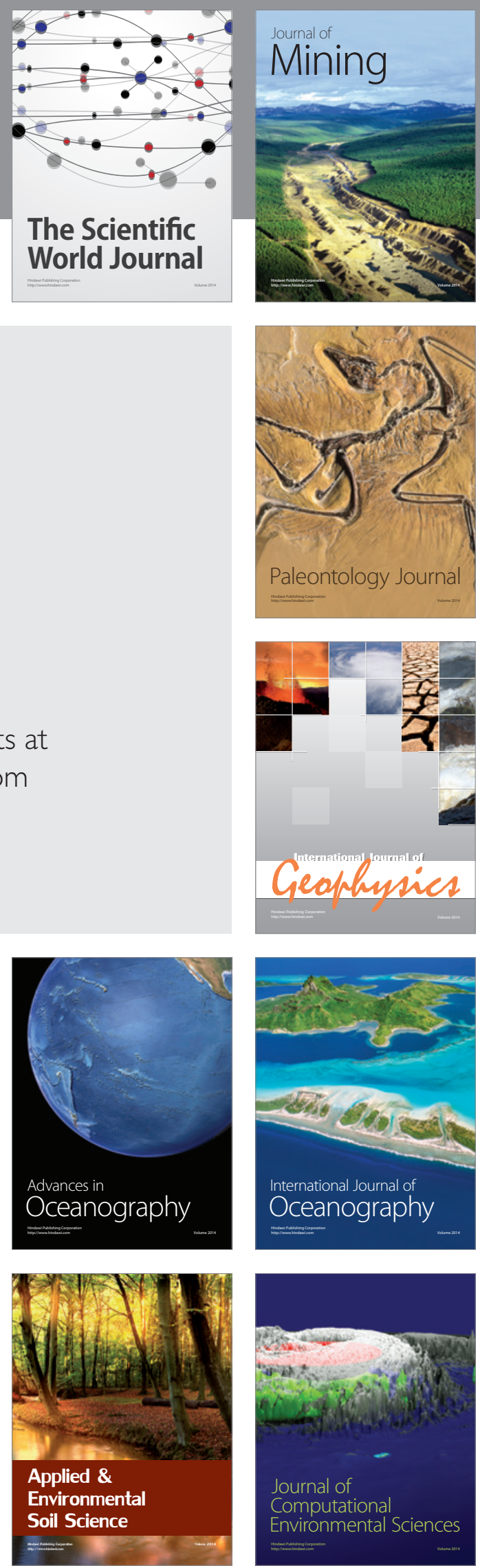